\title{
stabilisation d'une pente marine bi-couche par vibration profonde
}

\author{
par \\ A. Renouf \\ Ingénieur des T.P.E. \\ Subdivision Travaux du Port de Boulogne-sur-Mer \\ J.-P. lorio \\ Ingénieur civil des Ponts et Chaussées \\ Bureau d'Études - Entreprise SIF BACHY \\ G. Besançon \\ Ingénieur \\ Service Travaux - Entreprise SIF BACHY
}

\section{Introduction}

Le Service Maritime du Port de Boulogne-sur-Mer a procédé, en 1976, à la réalisation d'un terre-plein de 15 ha, par empire directe sur la mer.

Les fonds étaient constitués d'une épaisse couche de vase, sédiments apportés par les courants à l'intérieur du piège constitué par les digues.

A l'emplacement des travaux, la vase régnait de la cote $-13,00 \mathrm{CM}$ (rapportée au zéro des Cartes Marines) à la carte $-1,00 /-2,00 \mathrm{CM}$, soit légèrement en-dessous du niveau des plus basses mers $(0,00 \mathrm{CM})$.

Le terre-plein a été élevé à la cote moyenne $+11,00 \mathrm{CM}$ en remblayant hydrauliquement avec du sable prélevé sur des bancs au large du port.

Au niveau des digues d'enclôture, la vase a été draguée, et substituée par du sable clapé, pour constituer une assise solide à la digue.

Étant donné la profondeur du traitement, ce procédé n'était pas applicable à proximité d'ouvrages existants, sans mettre en cause leur stabilité propre.

A la fin des travaux est donc restée une brèche de $130 \mathrm{~m}$ dans la digue d'enclôture, remplacée par une pente naturelle de sable.

\section{Problèmes posés et solutions envisagées}

\subsection{Problèmes posés}

Le problème principal concernait l'édification de la digue d'enclôture, au-dessus d'une épaisse couche de vase, sans risquer une rupture par glissement d'ensemble de l'ouvrage en cours ou après construction.

La mise au point d'une technique économique pour la réalisation de la digue passait par l'exécution d'études de sols préalables, qui ont été effectuées en 1978.

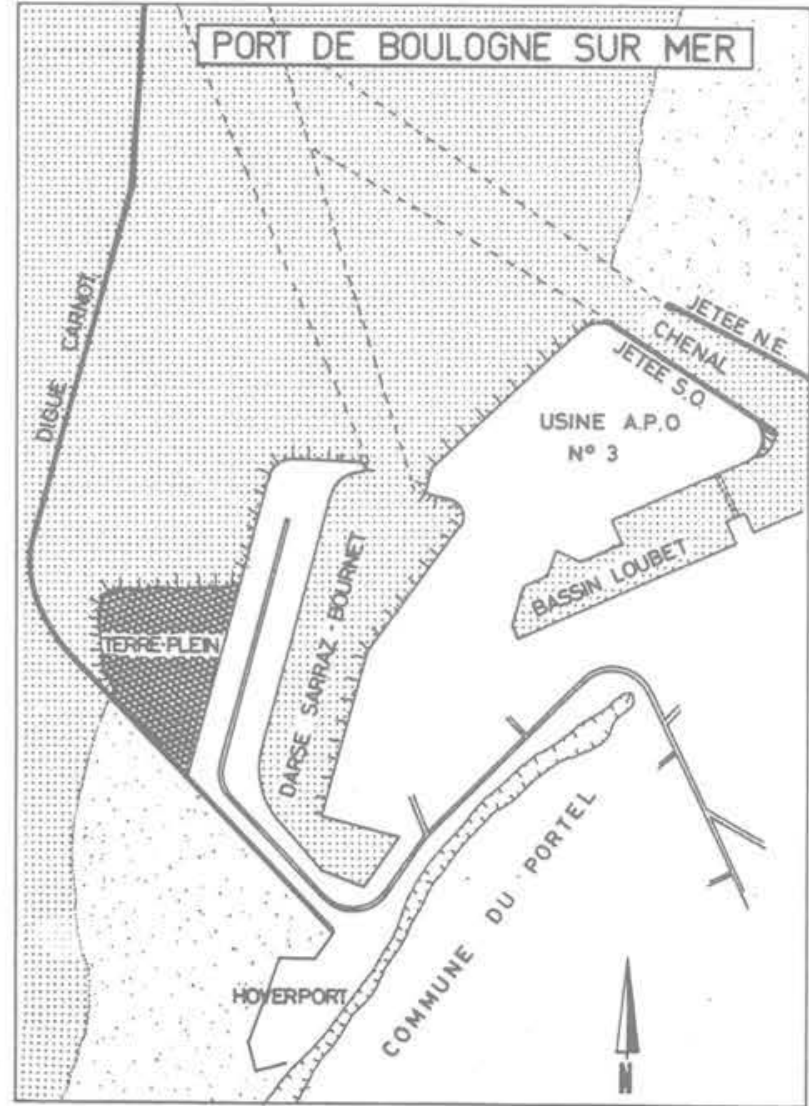

Fig. 1 Plan de situation du terre-plein 


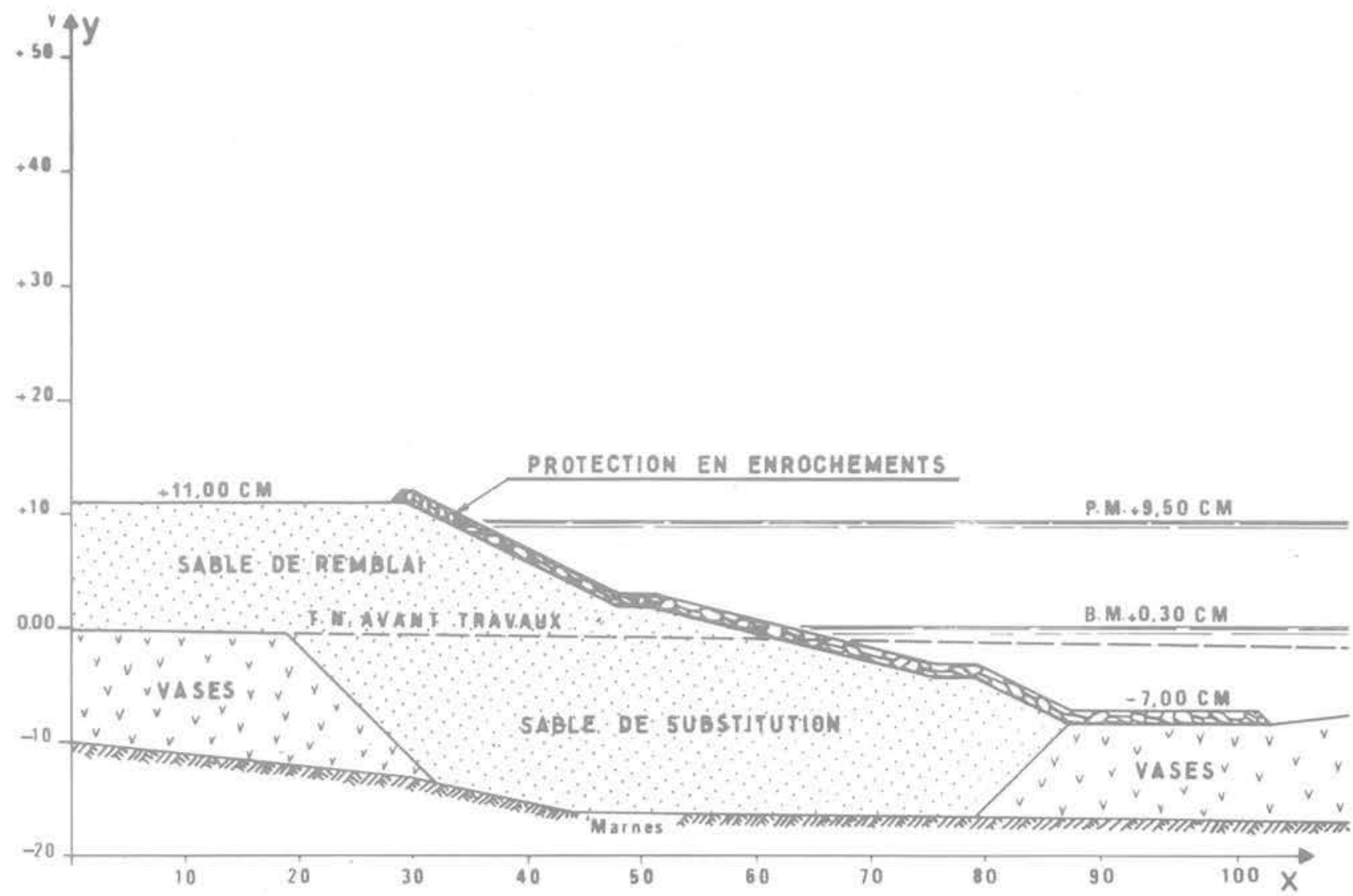

Fig. 2 Coupe-type de la limitation utilisée sur la façade Nord du terre-plein

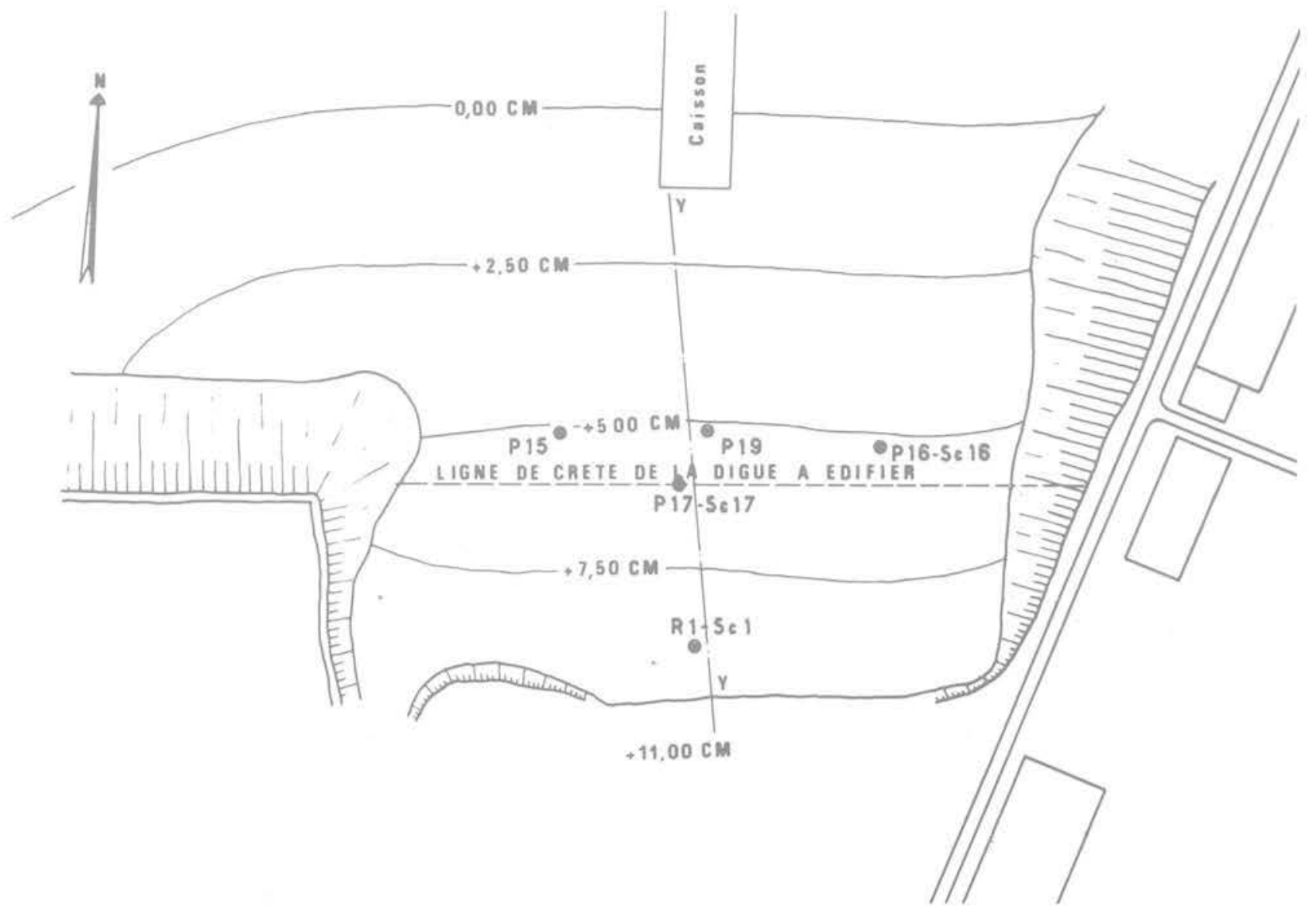

Fig. 3 Plan de la zone où la digue d'enclôture n'a pu être construite. A gauche, la limitation du nouveau terre-plein, à droi. te la limitation existante 


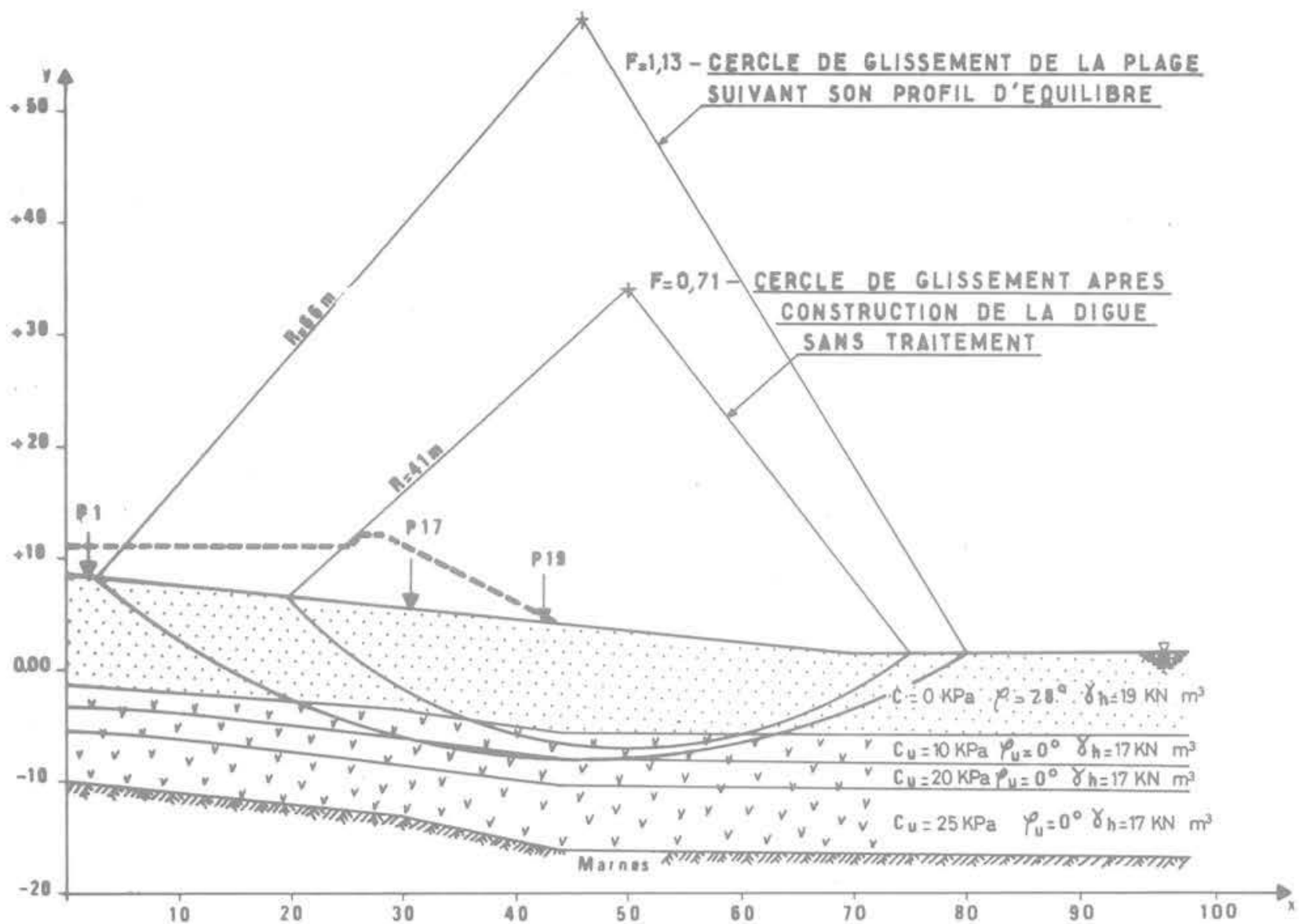

Fig. 4 Coupe géologique structurale suivant l'axe $Y Y$ '

\section{2 Études de sols}

Une campagne de sondage a été menée sous la surveillance du Bureau de Recherches Géologiques et Minières (B. R. G. M.).

Elle comprenait un forage d'identification, et des essais in situ : pénétromètre statique et scissomètre (voir implantation, fig. 3).
La reconnaissance a permis de dresser une coupe des terrains, confirmant la présence d'une couche de vase de 6 à $8 \mathrm{~m}$ d'épaisseur surmontée de 8 à $10 \mathrm{~m}$ de sable peu compact (fig, 4).

Les mesures effectuées sur place, et le résultat des essais en Laboratoire ont permis de retenir, pour les calculs de stabilité, les valeurs moyennes suivantes :

\begin{tabular}{|c|c|c|c|c|}
\hline Nature du sol & $\begin{array}{l}\text { Épaisseur des } \\
\text { couches }\end{array}$ & \multicolumn{3}{|c|}{ Caractéristiques } \\
\hline Sable & 8 à $10 \mathrm{~m}$ & \multicolumn{3}{|c|}{$\mathrm{C}=0 \mathrm{kPa} \phi=28^{\circ} \gamma \mathrm{h}=19 \mathrm{kN} / \mathrm{m}^{3}$} \\
\hline Vase & 6 à 8 m & \multicolumn{3}{|c|}{$\begin{array}{l}\mathrm{Cu}=\text { variant de } \\
10 \mathrm{kPa} \text { à } 25 \mathrm{kPa} \varphi \mathrm{u}=0^{\circ} \\
\text { avec la profondeur } \gamma \mathrm{h}=17 \mathrm{kN} / \mathrm{m}^{3}\end{array}$} \\
\hline \multicolumn{5}{|c|}{$\begin{array}{l}\text { Les calculs de stabilité ont été menés par la méthode } \\
\text { de Bishop, et les résultats ont été les suivants: }\end{array}$} \\
\hline \multirow{2}{*}{$\begin{array}{l}\text { Profil du } \\
\text { sol }\end{array}$} & \multicolumn{4}{|c|}{ Caractéristiques des cercles les plus défavorables } \\
\hline & \multicolumn{2}{|c|}{$\begin{array}{c}\text { Coordonnées du centre } \\
X\end{array}$} & Rayon (R) & $\begin{array}{l}\text { Coefficient } \\
\text { de sécurité (F) }\end{array}$ \\
\hline État actuel & 46 & 58 & $66 \mathrm{~m}$ & 1,13 \\
\hline $\begin{array}{l}\text { Après } \\
\text { construction } \\
\text { de la digue }\end{array}$ & 50 & 34 & $41 \mathrm{~m}$ & 0,71 \\
\hline
\end{tabular}


L'examen de ces résultats montre que l'édification de la digue n'est pas envisageable sans procéder à des améliorations, soit des caractéristiques géométriques de la digue, soit des caractéristiques mécaniques des sols.

\subsection{Solutions sans traitement profond}

A ce titre ont été envisagées deux solutions, correspondant à des techniques de coût réduit.

\subsubsection{Construction de la digue par étapes}

Sur la figure 5 est représenté (en grisé) l'ouvrage partiel dont la réalisation a été envisagée.

Cet ouvrage permettait de réaliser un préchargement des couches compressibles, la réalisation de l'ouvrage définitif étant subordonnée à l'attente de consolidation de la vase.

Cette solution a été abandonnée, car d'une part la stabilité de la digue seule était précaire d'autre part, I'accroissement de cohésion induit par le chargement aurait été limité: dans l'espace (sous l'emprise du projet seulement), et en valeur (inférieure à $10 \mathrm{kPa}$ ).

\begin{tabular}{|c|r|r|c|c|}
\hline \multirow{2}{*}{$\begin{array}{c}\text { Profil } \\
\text { du sol }\end{array}$} & \multicolumn{4}{|c|}{ Caractéristiques des cercles les plus défavorables } \\
\cline { 2 - 5 } & $\mathrm{X}$ & $\mathrm{Y}$ & $\mathrm{R}$ & $\mathrm{F}$ \\
\hline Digue partielle & 49 & 31 & $38 \mathrm{~m}$ & 0,92 \\
\hline
\end{tabular}

\subsubsection{Adjonction d'une banquette de pied}

Une seconde étude a été menée comprenant la réalisation de la digue avec adjonction de l'effet stabilisateur d'une banquette de pied (cf. fig. 5).

Les cercles de rupture les plus critiques avaient les caractéristiques suivantes :

\begin{tabular}{|l|c|c|c|c|c|}
\hline \multirow{2}{*}{$\begin{array}{c}\text { Profil } \\
\text { du sol }\end{array}$} & \multicolumn{5}{|c|}{ Caractéristiques des cercles les plus défavorables } \\
\cline { 2 - 6 } & $n^{\circ}$ & $X$ & $Y$ & $R$ & $F$ \\
\hline $\begin{array}{l}\text { Digue avec } \\
\text { banquette } \\
\text { de pied }\end{array}$ & 1 & 53 & 22 & 31 & 0,83 \\
& 3 & 50 & 34 & 43 & 0,80 \\
& 33 & 46 & 53 & 0,75 \\
\hline
\end{tabular}

Cette banquette a été réalisée en 1979, d'une part à cause de l'opportunité qui nous était offerte à l'époque de disposer de matériaux à faible coût, et d'autre part pour constituer une plate-forme de travail pour les traitements profonds dont nous voyions apparaître la nécessité.

\subsection{Solutions avec traitement profond}

Une consultation d'Entreprises spécialisées a été effectuée en 1979, avec pour objectif de proposer des solutions chiffrées pour l'édification de la digue.

En dehors des considérations de coût, les solutions présentées ont été examinées en tenant compte des contraintes liées au projet de construction d'un hangar sur les terre-pleins à construire : contraintes de délai (urgence des besoins en surface couverte) et contrainte supplémentaire pour la stabilité (surcharge de $60 \mathrm{kPa}$ à $10 \mathrm{~m}$ en arrière des limitations).
Les diverses solutions présentées peuvent se ranger dans quatre catégories principales, présentées au tableau 1.

\subsection{Marche}

Un marché a finalement été passé avec l'Entreprise SIF $\mathrm{BACHY}$ sur la base de la solution 3 , pour un montant de 2014000 F T.T.C., et une durée de trois mois.

Le marché stipulait que l'Entreprise devait vérifier par le calcul la tenue de la digue en examinant trois cas :

- un cas de chantier correspondant à une des phases de travaux : stabilité sous la charge apportée par une plate-forme à $+9,50$, après réalisation d'une première partie du traitement;

- deux cas correspondant à l'exploitation ultérieure, soit :

- stabilité sous la charge des remblais à leur cote 


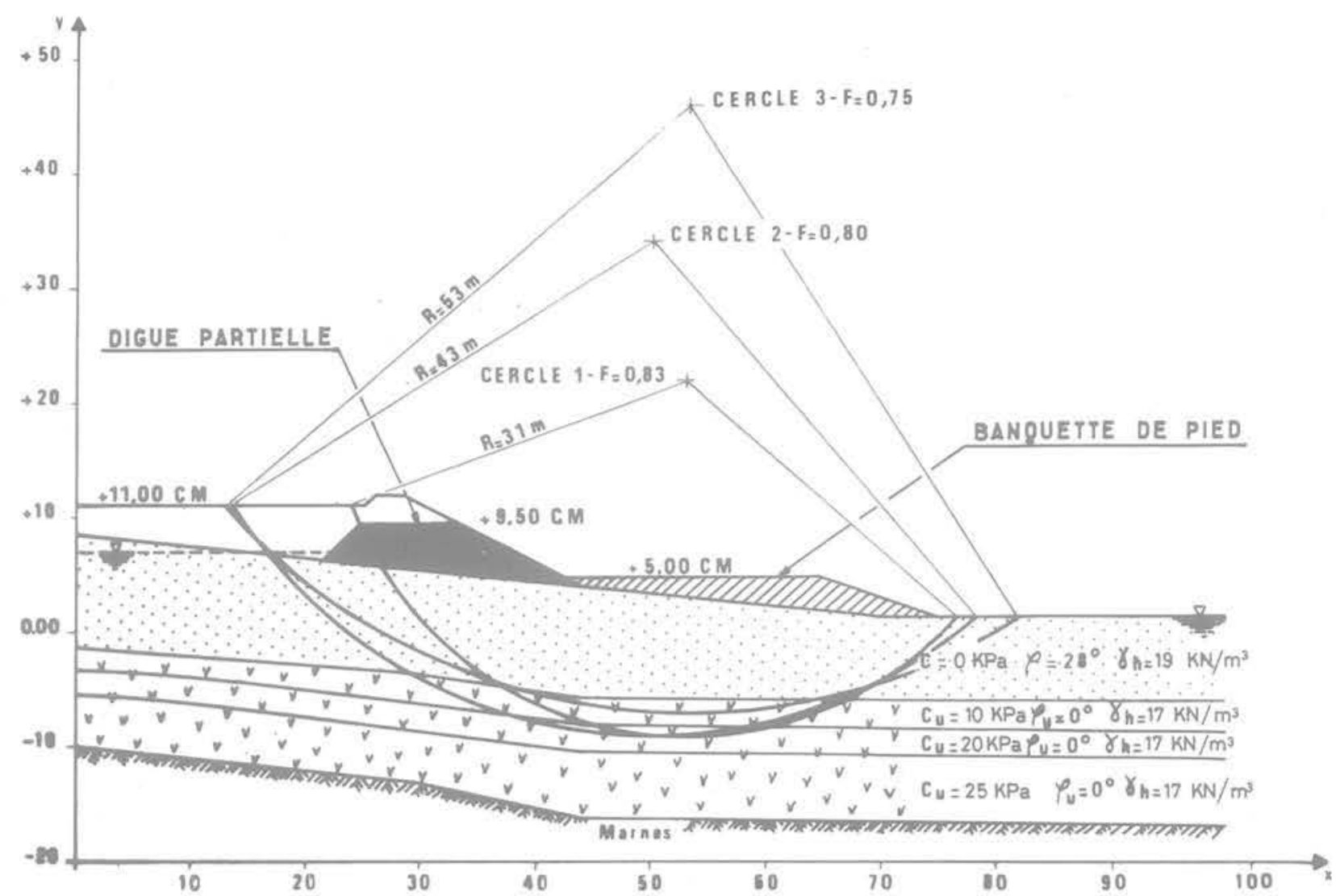

Fig. 5 Coupe des projets envisagés sans traitement profond. En grisé, la digue partielle. En hachuré, la banquette de pied

Tableau 1: Résultats de la consultation d'Entreprises spécialisées

\begin{tabular}{|c|c|c|c|}
\hline \multirow{2}{*}{$\begin{array}{l}\text { Désignation de la technique } \\
\text { proposée }\end{array}$} & \multirow{2}{*}{$\begin{array}{l}\text { Coût } \\
\text { (caleur mai } \\
\text { 1979) }\end{array}$} & \multicolumn{2}{|c|}{ Observations } \\
\hline & & Avantages & Inconvénients \\
\hline 1) Vibroflottation du sable & $\begin{array}{l}1500000 \text { pour } \\
4400 \mathrm{~m}^{2}\end{array}$ & Effet immédiat & $\begin{array}{l}\text { Rupture non circulaire } \\
\text { probable dans la vase }\end{array}$ \\
\hline 2) Drains (P.V.C.) & $\begin{array}{l}2500000 \text { pour } \\
5500 \mathrm{~m}^{2}\end{array}$ & Méthode éprouvée & $\begin{array}{l}\text { Délai important avant } \\
\text { prise de possession } \\
\text { des terrains }\end{array}$ \\
\hline $\begin{array}{l}\text { 3) Vibroflottation du sable } \\
\text { et colonnes ballastées } \\
\text { dans la vase }\end{array}$ & $\begin{array}{l}2100000 \text { pour } \\
5500 \mathrm{~m}^{2}\end{array}$ & $\begin{array}{l}\text { - Effet immédiat } \\
\text { - Sécurité } \\
\text { s'améliorant } \\
\text { dans le temps } \\
\text { (effet de drain) }\end{array}$ & $\begin{array}{l}\text { Amélioration difficile } \\
\text { à apprécier } \\
\text { par le calcul }\end{array}$ \\
\hline $\begin{array}{l}\text { 4) Clouage des terrains } \\
\text { avec des portiques } \\
\text { (H. E. A. } 1000 \text { espacés } \\
\text { de } 2,20 \mathrm{~m} \text { ) }\end{array}$ & 3000000 & Effet immédiat & $\begin{array}{l}\text { - Méthode nouvelle } \\
\text { - Calcul de la stabilité } \\
\text { des portiques difficile } \\
\text { - Problèmes sur } \\
\text { la portance de l'appui } \\
\text { arrière des portiques }\end{array}$ \\
\hline
\end{tabular}




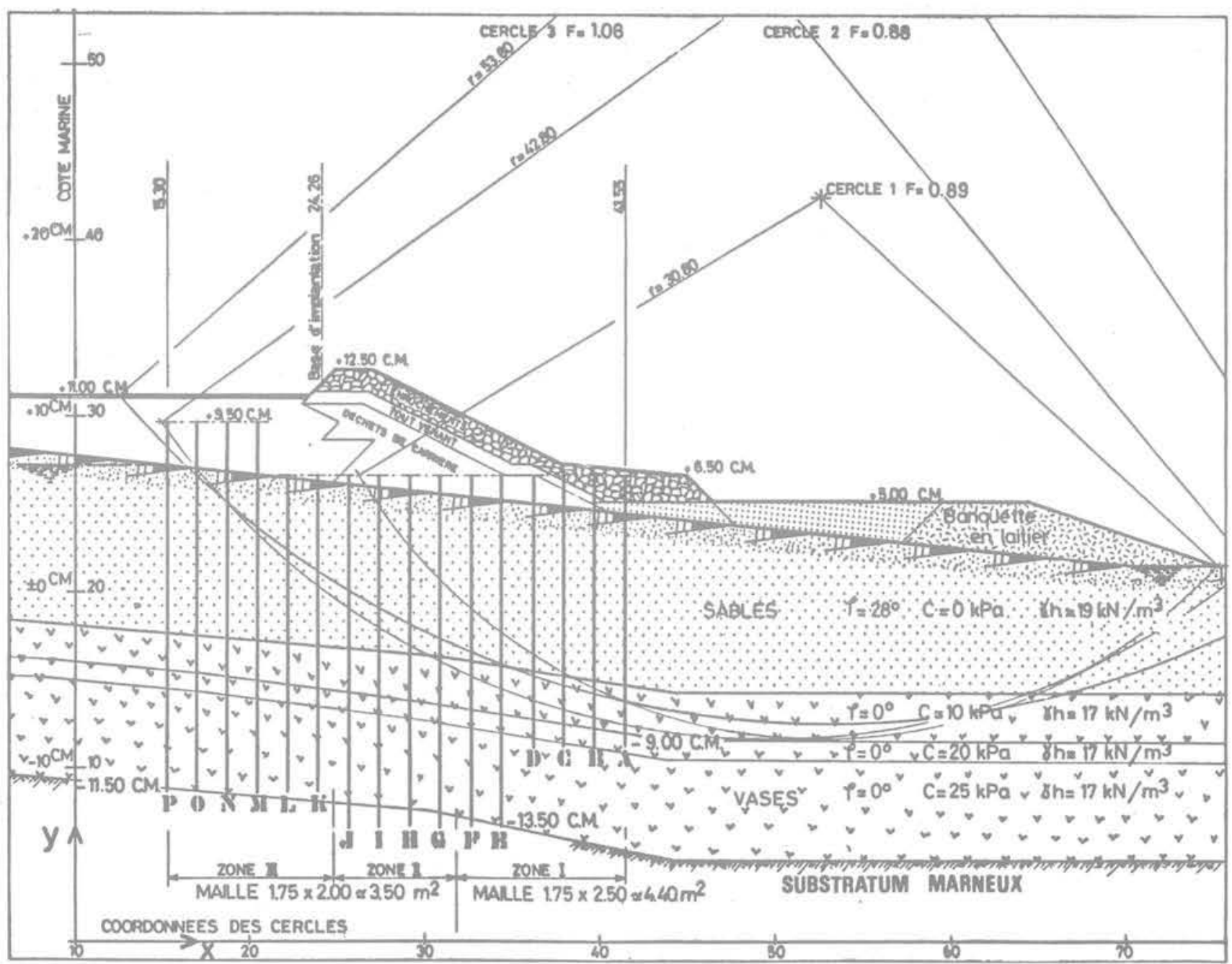

Fig. 6 Implantation et maillage des colonnes ballastées

définitive, appliquée deux mois après la fin des travaux de traitement de sols;

- stabilité sous la surcharge de $60 \mathrm{kPa}$, appliquée six mois après la fin des travaux de traitement de sols.

\section{Calculs}

Les calculs ont été menés en deux temps :

- dimensionnement du maillage des colonnes en fonction des charges verticales du remblai et de la force portante des colonnes;

- vérification de la stabilité au glissement circulaire en appréciant l'accroissement de résistance apporté par le traitement du terrain.

\subsection{Rappels sur le procédé de traitement de sols par vibration profonde}

Bien qu'utilisant un matériel identique, le procédé de vibration profonde comporte deux techniques fondamentalement différentes :

- vibroflottation pour traitement des sols non cohérents (sables);

- colonnes ballastées pour traitement des sols cohérents (argile).
Dans le cas présent de Boulogne-sur-Mer, il convenait de traiter la couche de sable lâche des remblais hydrauliques par vibroflottation, et d'exécuter des colonnes ballastées dans l'horizon des vases molles.

Nous n'insisterons pas sur la technique de vibroflottation qui est extrêmement classique, et qui a fait l'objet de nombreuses publications françaises et étrangères.

Par contre, il convient de rappeler les principes de fonctionnement des colonnes ballastées, étant donné que le problème de stabilité générale ne pouvait être réglé que par un traitement judicieux de la couche de vases molles, c'est-à-dire par le dimensionnement à adopter pour le dispositif de colonnes ballastées.

Stabilité des colonnes

a) Concentration de charge

La colonne ballastée revient à opérer une substitution ponctuelle dans la couche de mauvaises caractéristiques mécaniques, et constitue un point dur dont le module de déformation est nettement plus élevé que celui du sol encaissant.

L'observation montre que, sous la charge induite par l'ouvrage, les tassements sont identiques pour la colonne et le sol ambiant. II en résulte une concentration de charge sur la colonne ballastée. 


\section{REMBLAI ( surcharge)}

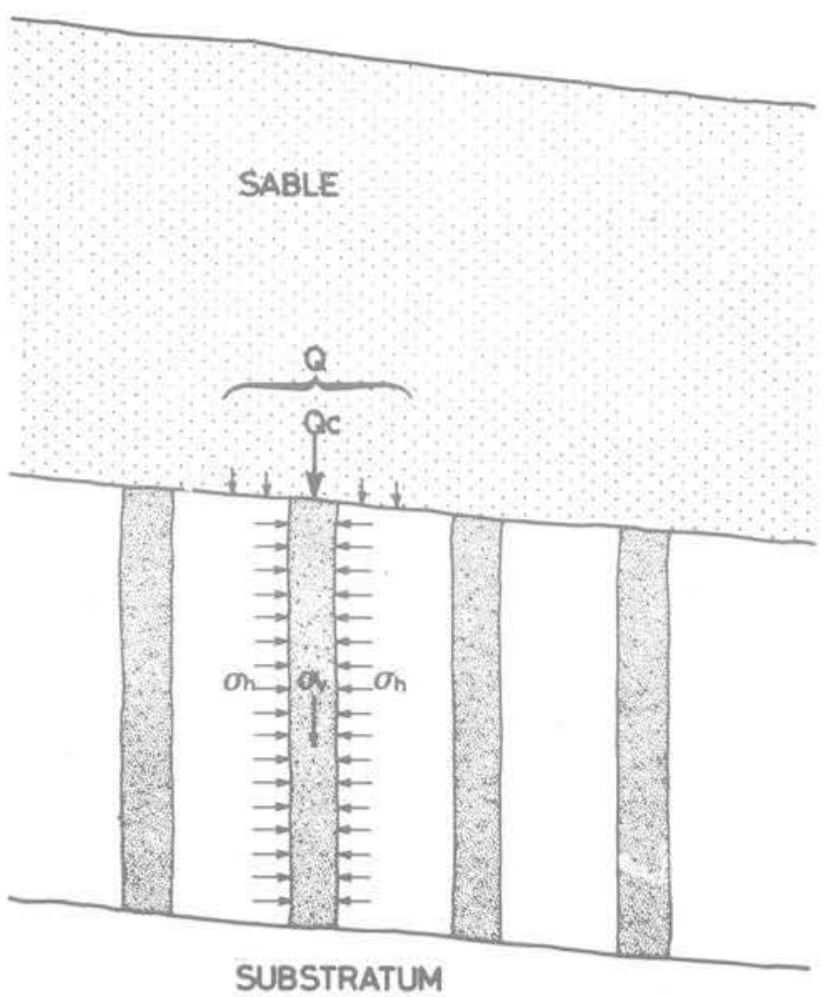

Fig. 7 Schéma de comportement des colonnes ballastées

Le premier calcul consistera donc à déterminer, par un calcul hyperstatique, le pourcentage de charge repris par les colonnes ballastées; dans une certaine mesure, on peut assimiler cette situation à une stabilité à court terme.

\section{b) Rôle de pseudo-pieu}

La concentration de charge peut devenir excessive. Or, les colonnes ballastées constituent des éléments de transition à caractéristiques mécaniques élevées, et permettent de reporter les charges sur un horizon sous-jacent. On peut admettre comme deuxième mode de calcul que ces pseudo-pieux supportent toute la surcharge du remblai.

Le mécanisme de transfert peut être assimilé à celui d'un échantillon pulvérulent placé dans l'appareil triaxial, car la colonne ballastée n'existe que grâce à la réaction latérale qui peut être fournie par le sol encaissant.

Cette réaction latérale correspond, à rupture, à la pression limite mesurée par l'essai pressiométrique, et peut d'ailleurs être donnée par corrélation, par tout autre essai in situ tel que pénétromètre ou scissomètre.

Le deuxième calcul consistera donc à déterminer la force portante admissible des colonnes ballastées, et par conséquent, le maillage du traitement en fonction des charges induites par l'ouvrage.

\section{Effet du drainage}

La colonne ballastée étant constituée par un matériau d'apport graveleux non cohérent, il en résulte un effet de drainage de la couche de vase.
II s'ensuit une amélioration des caractéristiques mécaniques du sol ambiant, par dissipation des pressions interstitielles. Cette amélioration à long terme, ne peut pas être prise en compte dans le dimensionnement du traitement, tous les calculs devant être réalisés à court terme.

Par contre, cet effet drainant apporte une sécurité supplémentaire très intéressante car, par application de la théorie de consolidation radiale de Barron, comme on la trouve par exemple dans Magnan (1977), et en assimilant les colonnes ballastées à de gros drains de sable, on constate que $90 \%$ de la consolidation des vases sera obtenu dans un délai très rapide (quelques mois).

\subsection{Dimensionnement du réseau de colonnes ballastées : force portante et maillage}

\subsubsection{Stabilité à court terme}

Le premier calcul a été conduit en assimilant la colonne ballastée à un ressort, et en recherchant le pourcentage de charge qui pourrait être repris par la colonne.

Ce calcul repose sur deux constatations résultant de nombreux essais et calculs effectués tant en France qu'à l'étranger :

- le tassement à la surface du terrain naturel est identique à celui mesuré en tête des colonnes (d'après J. Vautrain, 1980);

- le module de déformation dans la colonne ballastée est au moins 10 fois supérieur à celui du sol encaissant, sans toutefois pouvoir excéder une valeur de $100 \mathrm{MPa}$.

Extrait de l'article : «Stabilisation d'une pente marine bi-couche par vibration profonde."

En écrivant l'égalité des déformations, on trouve la charge reprise par la colonne:

$$
Q_{c} / Q=10 \times S_{c} /\left(10 \times S_{c}+S_{s}\right)
$$

formule dans laquelle :

$Q_{c}=$ charge reprise par la colonne,

$\mathrm{Q}=$ charge totale,

$\mathrm{S}_{\mathrm{c}}=$ surface de la colonne,

$\mathrm{S}_{\mathrm{s}}=$ surface du sol ambiant (surface maillage, surface colonne).

On verra au paragraphe suivant (\$ 3.2.2) que les charges transmises aux colonnes sont nettement inférieures à leur limite de rupture.

Avec un module de déformation des vases $E_{s}=1 \mathrm{MPa}$ qui résulte du rapport de sols, et avec les maillages envisagés (fig. 6 ) on arrive aux résultats suivants :

\begin{tabular}{|c|c|c|c|}
\hline Zone de traitement & I & II & III \\
\hline Maillage du traitement & $\begin{array}{r}1,75 \times 2,50 \\
\# 4,40 \mathrm{~m}^{2}\end{array}$ & $\begin{array}{r}1,75 \times 2 \\
=3,50 \mathrm{~m}^{2}\end{array}$ & $\begin{array}{c}1,75 \times 2 \\
=3,50 \mathrm{~m}^{2}\end{array}$ \\
\hline $\mathrm{Q}_{\mathrm{c}} / \mathrm{Q}$ & 0,75 & 0,80 & 0,80 \\
\hline $\begin{array}{c}\text { Coefficient de sécurité } \\
\text { sur la portance des } \\
\text { colonnes à court terme }\end{array}$ & 1,69 & 1,29 & 1,57 \\
\hline
\end{tabular}


On constate ainsi que le dimensionnement du projet est parfaitement admissible à court terme.

\subsubsection{Pseudo-pieu à rupture}

Le deuxième calcul a été conduit en assimilant la colonne ballastée à l'éprouvette de matériau pulvérulent placée dans l'appareil triaxial, c'est-à-dire par application de la formule :

$$
\sigma v=P \ell \times(1+\sin \phi) /(1-\sin \phi),
$$

dans laquelle:

$\phi=38^{\circ}$ valeur minimum de l'angle de frottement interne de la colonne,

$\mathrm{PI}=60 \mathrm{kPa}$, valeur de la pression limite des vases ressortant du rapport de sols B. R. G.M.

Cette valeur de pression limite correspond aux valeurs les plus faibles mesurées en tête de la couche de vase, soit au scissomètre $\left(\mathrm{C}_{\mathrm{u}}=10 \mathrm{kPa}\right)$, soit au pénétromètre statique $\left(R_{p} \# 0,2 \mathrm{MPa}\right)$.

Nous avons pris par ailleurs les hypothèses suivantes :

- section droite de la colonne de $1 \mathrm{~m}^{2}$, ce qui correspond en pratique à une incorporation de matériau d'apport foisonné de $1,5 \mathrm{~m}^{3} / \mathrm{ml}$;

- totalité des charges reprise par les colonnes.

Sur les bases ci-dessus, la capacité portante d'une colonne ballastée, à rupture, a été estimée à $250 \mathrm{kN}$.
Cet état de contraintes étant sujet à variation dans le temps, nous avons envisagé trois hypothèses traduisant chacune un état limite susceptible d'exister au sein des colonnes.

\section{Hypothèse $A$}

Pendant et immédiatement après le chargement, l'étreinte latérale des colonnes est égale à la pression limite du sol ambiant.

Les contraintes principales dans les colonnes sont alors :

$$
\begin{aligned}
& \sigma \mathrm{h}=\mathrm{Pl}, \\
& \sigma \mathrm{V}=\mathrm{Q} / \mathrm{S}_{\mathrm{c}} \text { avec une limite supérieure égale à } \mathrm{j} \times \mathrm{PI},
\end{aligned}
$$

$(j=(1+\sin \phi) /(1-\sin \phi)$, rapport limite des contraintes principales à la rupture, $j=4,20$ pour $\phi=38^{\circ}$ ).

Dans notre cas, nous prendrons $\sigma \mathrm{v}=\mathrm{j} \times \mathrm{PI}$, puisque les calculs ont montré que les colonnes seraient à la limite de leur charge admissible.

Dans cette hypothèse, les vases sont prises en compte avec leurs caractéristiques à court terme, soit :

$$
\phi_{\mathrm{u}}=0^{\circ}, \quad \mathrm{C}_{\mathrm{u}}=10 \mathrm{kPa} \text {. }
$$

\section{Hypothèses B1 et B2}

Un certain temps après le chargement, les colonnes subissent une relaxation, et on assiste à une redistribution des charges de remblai.

Les résultats obtenus sont résumés dans le tableau ci-dessous:

\begin{tabular}{|c|c|c|c|}
\hline Zone de traitement & I & II & III \\
\hline Maillage du traitement & $\begin{array}{c}1,75 \times 2,50 \\
\# 4,40 \mathrm{~m}^{2}\end{array}$ & $\begin{array}{c}1,75 \times 2 \\
=3,50 \mathrm{~m}^{2}\end{array}$ & $\begin{array}{c}1,75 \times 2 \\
=3,50 \mathrm{~m}^{2}\end{array}$ \\
\hline Charge du terre-plein & $45 \mathrm{kPa}$ & $69 \mathrm{kPa}$ & $57 \mathrm{kPa}$ \\
\hline $\begin{array}{c}\text { Coefficient de sécurité } \\
\text { sur la portance } \\
\text { des colonnes }\end{array}$ & 1,27 & 1,04 & 1,25 \\
\hline
\end{tabular}

Dans ce cas particulier de traitement de sols, il est parfaitement admissible de faire travailler les colonnes avec un coefficient de sécurité légèrement supérieur à 1 .

En effet, d'une part la répartition des charges envisagée pour le court terme au paragraphe précédent (\$ 3.2.1) continuera à exister, et d'autre part, à plus long terme, les vases se consolideront et fourniront une étreinte latérale supérieure à celle qui a été prise en compte ici.

3.3 Vérification de la stabilité générale au glissement circulaire

\subsubsection{Résistance au cisaillement des colonnes} ballastées. Hypothèses

Le calcul de la résistance au cisaillement apportée par les colonnes est délicat du fait que le sol traité constitue un matériau anisotrope, et que cette résistance dépend de l'état de contraintes des colonnes.
A long terme, la charge reprise par les colonnes est un pourcentage du poids des terres, ce pourcentage étant compris entre 1 (hypothèse B2: la charge du terre-plein est uniformément répartie sur les colonnes et le terrain naturel), et $\mathrm{j}$ (hypothèse B1).

Les contraintes principales dans les colonnes sont alors :

\begin{tabular}{|c|c|}
\hline Hypothèse B1 & Hypothèse B2 \\
\hline$\sigma h=\gamma Z$ & $\sigma h=\gamma Z$ \\
$\sigma v=j \times \gamma Z$ & $\sigma v=\gamma Z$ \\
\hline
\end{tabular}

Dans ce cas, les vases sont prises en compte avec des caractéristiques améliorées : $\mathrm{C}=20 \mathrm{kPa}$, pour tenir compte de leur consolidation.

Cette amélioration de la cohésion est attestée par les mesures de cohésion après traitement effectuées au scissomètre (J. Vautrain, 1980). 


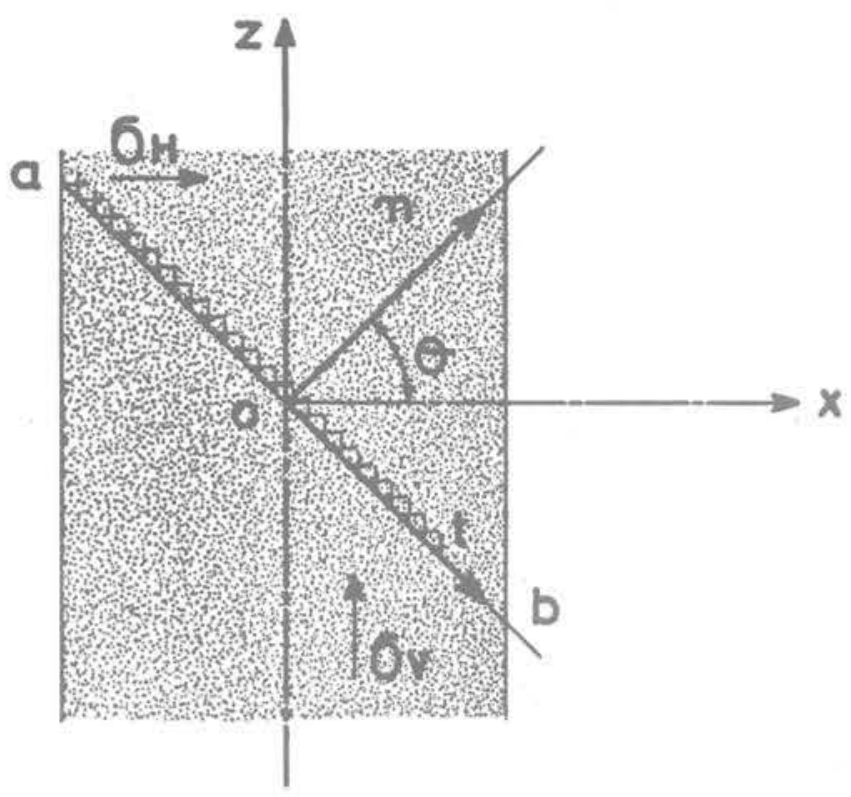

Fig. 8 Distribution des contraintes dans la collonne ballastée

Calcul de la résistance au cisaillement à partir de $(\sigma h, \sigma v)$

Le plan de rupture (ab) coupant l'axe de la colonne en $O$ à la profondeur $Z$, est défini par l'angle $\theta$ de sa normale avec l'axe horizontal $\overrightarrow{\mathrm{Ox}}$ (fig. 8).

La contrainte normale $n$ et la contrainte tangentielle $t$ au plan de rupture se déduisent des contraintes principales $\sigma \mathrm{h}$ et $\sigma \mathrm{v}$ par les relations:

$$
\begin{aligned}
& n=\sigma h \cos ^{2} \theta+\sigma v \sin ^{2} \theta \\
& t=(\sigma v-\sigma h) \sin \theta \cos \theta .
\end{aligned}
$$

Connaissant $(n, t)$ on obtient la contrainte de résistance au cisaillement :

$$
\tau \mathrm{C}=\mathrm{n} \operatorname{tg} \phi-\mathrm{t}
$$

formule dans laquelle la contrainte tangentielle $t$ est exprimée en valeur algébrique suivant l'inclinaison $\theta$ et le signe de $(\sigma v-\sigma h)$.

D'où on déduit la force de résistance au cisaillement mobilisable dans la colonne suivant l'inclinaison $\theta$ :

$$
\mathrm{Tc}=\tau \mathrm{c} \times \mathrm{Sc} / \mathrm{Sin} \theta=(\mathrm{n} \operatorname{tg} \phi-\mathrm{t}) \times \mathrm{Sc} / \sin \theta .
$$

\subsubsection{Déroulement du calcul. Résultats}

La stabilité générale au glissement circulaire a été vérifiée suivant la méthode de Fellenius.

Pour les trois cercles étudiés, le calcul est fait en deux étapes :

a) Calcul du coefficient de sécurité sans colonnes ballastées avec les caractéristiques du terrain non traité

Le coefficient de sécurité $F_{s}$ est défini comme d'habitude par le rapport du moment résistant au moment moteur.

Le moment résistant est calculé à partir de la résistance au cisaillement $t_{1}=n$ tg $\phi$ dans les sables et $\mathrm{t}_{2}=\mathrm{C}_{u}$ dans les vases.

b) Calcul du coefficient de sécurité prenant en compte le traitement
On admet toujours la même définition de $\mathrm{F}_{\mathrm{s}}$ mais, cette fois-ci, le moment résistant est calculé à partir de $t_{1}=n$ tg $\phi_{1}$ dans les sables $\left(\phi_{1}\right.$ angle de frottement amélioré), $\mathrm{t}_{2}=\mathrm{C}$ dans les vases ( $\mathrm{C}$ cohésion améliorée, hypothèses $\mathrm{B} 1$ et $\mathrm{B} 2$ ) et $\tau_{\mathrm{c}}$ résistance au cisaillement des colonnes définie précédemment (hypothèses A, B1 et B2) chacune de ces résistances au cisaillement étant affectée des aires correspondantes.

\section{Résultats}

Les résultats de l'étude effectuée sur ordinateur à l'aide du programme Cerglis de Sif Bachy figurent dans le tableau ci-dessous :

\begin{tabular}{|l|c|c|c|}
\hline Cercle critique $\mathrm{n}^{\circ}$ & 1 & 2 & 3 \\
\hline $\mathrm{F}_{\mathrm{s}}$ (sans traitement) & 0,89 & 0,88 & 10,6 \\
\hline $\mathrm{F}_{\mathrm{s}}$ (après traitement) & & & \\
Hypothèse A & 1,61 & 1,78 & 1,95 \\
Hypothèse B1 & 1,40 & 2,09 & 2,08 \\
Hypothèse B2 & 1,32 & 1,53 & 1,61 \\
\hline
\end{tabular}

Le coefficient de sécurité le plus critique après traitement est égal à 1,61 à court terme (hypothèse A) et est compris entre 1,32 et 1,40 à long terme (hypothèses B1 et B2).

L'augmentation relative du coefficient de sécurité à long terme est supérieure à $48 \%$ pour les trois cercles étudiés.

II peut être noté que le traitement des sables supérieurs (augmentation de l'angle de frottement par vibroflottation) entre pour un part notable dans la stabilité à court terme. Par contre, suivant les hypothèses B1 et B2, le traitement des sables perd son caractère prédominant par suite de l'homogénéisation relative des sols traités.

\section{Description des travaux et contrôles}

\subsection{Phasage des travaux}

La stabilité précaire de la pente existante avant travaux, bien que confortée par la réalisation de la banquette de pied, a nécessité de faire commencer le traitement sur une plate-forme épousant au plus près le talus naturel existant.

Le phasage suivant a donc été retenu pour l'exécution des travaux:

Phase 1 : exécution du traitement de sols (files $\mathrm{A}$ à $\mathrm{L}$ ) à partir d'une plate-forme à $+6,50 \mathrm{CM}$ (submergée à chaque marée).

Phase 2: réalisation par l'Entreprise retenue pour la construction de la digue d'une première tranche de remblais à $+9,50 \mathrm{CM}$, bénéficiant de l'amélioration des sols provoquée par le premier traitement.

Phase 3 : réalisation de la dernière partie du traitement de sols à partir de cette plate-forme.

Ce phasage a été mis au point dans le souci de limiter le temps de travail à la marée, plus long et plus coûteux. II présente d'autre part l'avantage d'imbriquer les travaux de traitement et de remblaiement, permettant de réduire d'autant le délai de mise à disposition de la plate-forme définitive. 


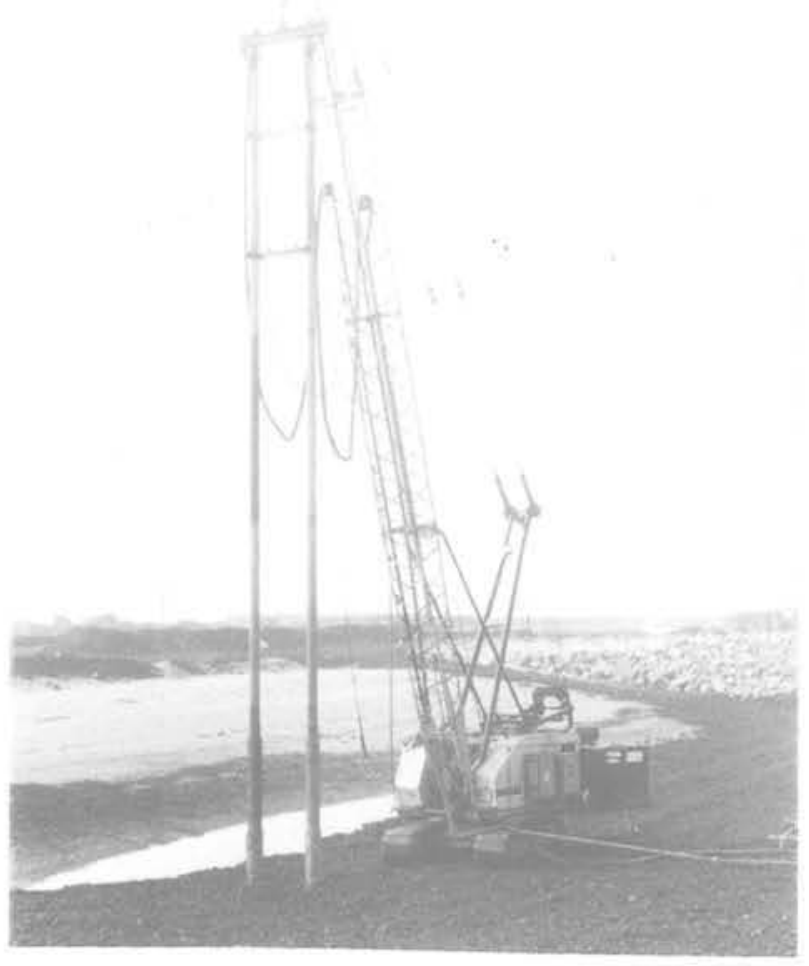

Fig. 9 Vibreurs couplés en position de travail

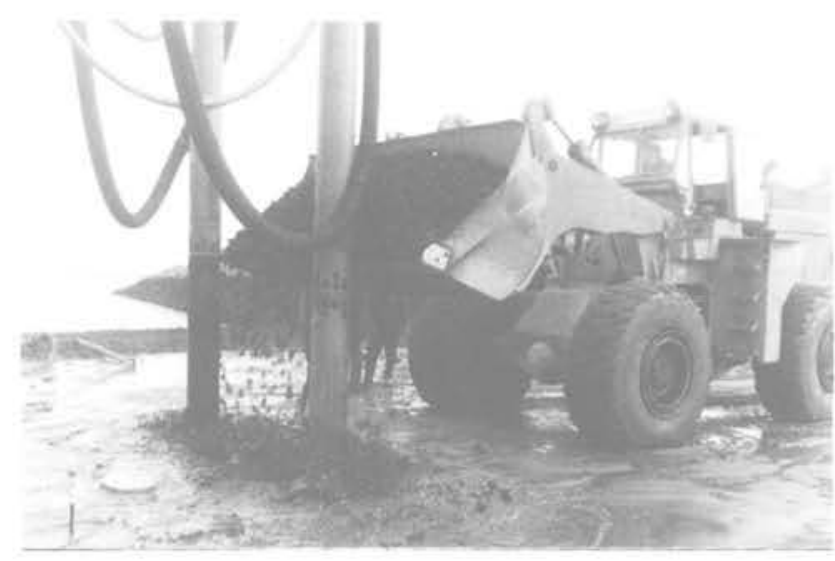

Fig. 10 Mise en place du matériau graveleux dans la colonne ballastée

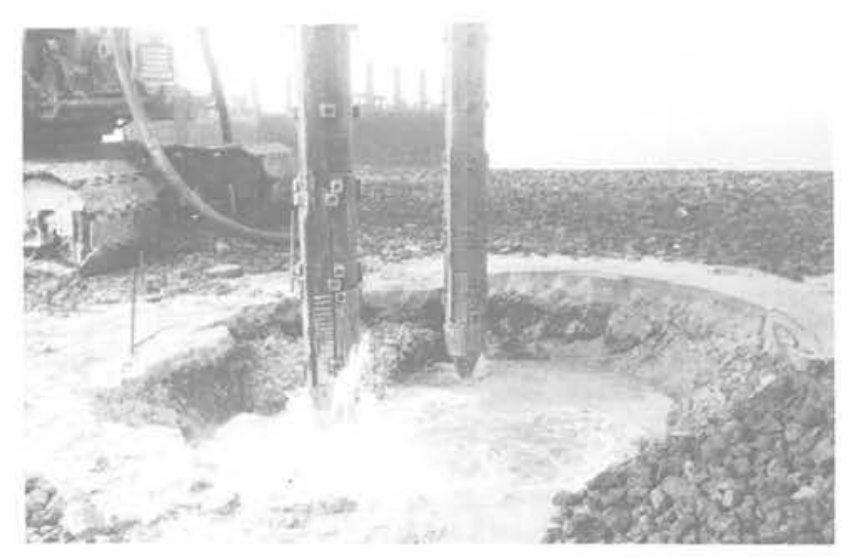

Fig. 11 Affaissement de la plate-forme en cours de fonçage

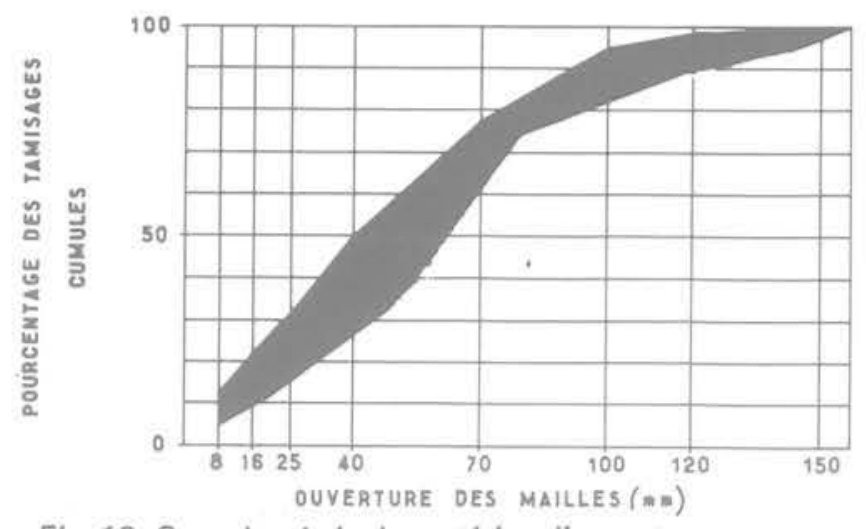

Fig. 12 Granulométrie du matériau d'apport

\subsection{Mode d'exécution des travaux}

L'exécution du traitement à partir de la plate-forme à $+6,50 \mathrm{CM}$ nécessitait de replier le matériel à chaque marée, limitant le temps de travail effectif à 5 ou 8 heures par marée (suivant l'amplitude de celle-ci).

Chaque marée ne permettait d'effectuer qu'un nombre restreint de colonnes (entre 8 à 10 colonnes).

Pour satisfaire à la contrainte de délai, l'Entreprise a mis en ceuvre des moyens puissants :

- grue Koering 605 équipée avec flèche de $27 \mathrm{~m}$ et supportant deux équipements de vibroflottation de 22,50 $\mathrm{m}$ de longueur;

- groupe électrogène de 275 KVA supporté par la machine;

- station de pompage en mer prévue pour débiter $200 \mathrm{~m}^{3} / \mathrm{h}$ sous 9 bars.

L'ensemble des travaux a été réalisé du 9 octobre au 20 décembre 1979, en travaillant une moyenne de 9 marées par semaine, avec deux équipes se relayant.

II à été réalisé $11500 \mathrm{ml}$ de traitement, avec des profondeurs de point de compactage variant de 15 à $22 \mathrm{~m}$.

Pour la réalisation des colonnes, il a été utilisé plus de 10000 t de matériau d'apport (tout-venant concassé de laitier de granulométrie 0 à $80 \mathrm{~mm}$ ).

Sur l'épaisseur de la couche de sable, seul un apport de sable a été nécessaire pour remplir les colonnes lors de la vibroflottation.

\subsection{Contrôles sur chantier}

La rapidité d'exécution des travaux a empêché de procéder à l'instrumentation des terrains, ce qui aurait permis de suivre in situ l'évolution des caractéristiques des sols.

Les contrôles ont donc porté sur les paramètres qu'il était possible de suivre en cours de chantier ;

- niveaux supérieurs et inférieurs de la vase.

L'interface sable-vase était nettement reconnaissable sur chantier par la variation de couleur de l'eau remontant à la surface. Les niveaux pris en compte pour les calculs ont pu ainsi être vérifiés de manière incontestable.

- taux d'incorporation du matériau d'apport.

II a pu être vérifié, en procédant à des essais d'information, que le taux d'incorporation, ramené au mètre linéaire de colonne, variait sur la hauteur de celle-ci. 
Cette variation est normale et exprime que la colonne s'expanse suivant un diamètre dont la valeur est fonction des caractéristiques du milieu ambiant.

Le graphique ci-dessous représente la variation du taux d'incorporation $\mathrm{dQ} / \mathrm{dh}$ en fonction de la hauteur (fig. 13).

L'application de cette loi de variation, établie à partir de valeurs résultant de l'expérience de l'Entreprise, mène à un taux moyen égal à $1,44 \mathrm{~m}^{3} / \mathrm{ml}$.

De nombreuses vérifications effectuées sur le chantier ont fait apparaître un taux moyen de $1,65 \mathrm{~m}^{3} / \mathrm{ml}$, valeur sensiblement supérieure à celle prise en compte dans les hypothèses de calcul.

\section{Conclusion}

Le traitement de sols par vibration profonde est une technique extrêmement intéressante dans le cas de "stabilisation de sols très compressibles".

Dans le cas bien précis de stabilisation avec cercles de grand glissement, une méthode originale de dimensionnement a été utilisée, qui fait l'objet de la présente communication.

Cette méthode, d'application aisée, présente un intérêt certain pour le dimensionnement des projets, en ce sens qu'elle traduit le comportement réel des colonnes ballastées, dont la résistance au cisaillement dépend de l'état de contraintes auquel elles sont soumises.

Elle fait largement appel à des concepts nouveaux sur le fonctionnement des colonnes ballastées, dont l'état de contraintes est examiné séparément à court terme (en contraintes totales) et à long terme (après dissipation des pressions interstitielles).

On s'apperçoit que, bien qu'à long terme la capacité portante des colonnes soit diminuée et qu'une relaxation ait lieu dans les vases, la redistribution des charges et la consolidation qui en résulte améliore le coefficient de sécurité de l'ouvrage, aussi bien vis-à-vis de la force portante que vis-à-vis de la stabilité générale.

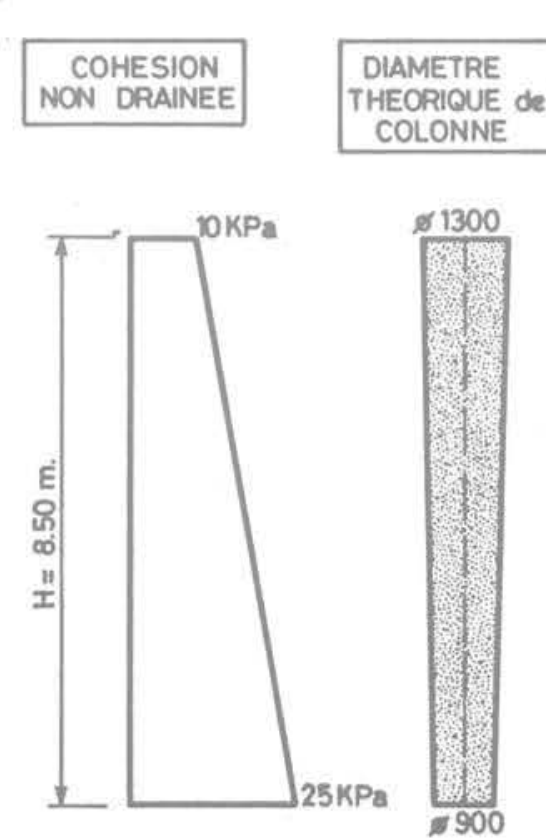

Cette technique n'est cependant pas utilisable en toutes circonstances, puisque la relaxation des colonnes envisagées dans le calcul s'accompagne obligatoirement de tassements. Ces tassements sont compatibles avec des structures déformables (remblais par exemple) ce qui était le cas à Boulogne-sur-Mer.

\section{Référence bibliographique}

M. Cassan (1974). «Etude des fondations du viaduc de Cagnes-sur-Mer, sur l'autoroute A8. " "Chantiers de France $» 1974$.

D.T.U. $n^{\circ} 13.2$ (1978), «Travaux de fondations profondes pour le bâtiment.» Chapitre VIII colonnes ballastées, juin 1978 .

K. Kirsch (1977). « Baugrundverbesserung unter Verwendung Von Tiefenrüttlern (Kurzfassung), „ Vortrag zum Seminar "Baugrundverbesserungen" and der Akademie Wuppertal im, mai 1977.

J.-P. Magnan (1977). « Remblais sur sols compressibles. "Séminaire A. A. E. N.P. C. Chapitre VI, étude des tassements, Paris, novembre 1977, pages 97-99.

M. Morgenthaler, Cambou et G. Sanglerat (1978). * Colonnes ballastées. Essais de chargement et calculs par la méthode des éléments finis. " Revue Française de Géotechnique $n^{\circ} 5$, novembre 1978, pages 41-55.

G. Pilot et al. (1977). « Méthodes d'amélioration des propriétés géotechniques des argiles molles. " International Symposium on Soft Clay, juillet 1977, Bangkok.

D. Remont et G. Besançon (1979). "Traitement des sols par vibroflottation et colonnes ballastées. Réalisation des fondations du complexe résidentiel de Gol E Schemal à Tchalus en Iran. " Travaux, novembre 1979, pages 30-36.

J. Vautrain (1977). « Mur en terre armée sur colonnes ballastées. " International Symposium on Soft Clay, juillet 1977, Bangkok.

J. Vautrain (1980). «Comportement et dimensionnement des colonnes ballastées. " Revue Française de Géotechnique $n^{\circ} 11$, mai 1980, pages 59-73. 